\title{
Front Matter: Volume 6690
}

, "Front Matter: Volume 6690," Proc. SPIE 6690, Focal Plane Arrays for Space Telescopes III, 669001 (26 October 2007); doi: 10.1117/12.773440

SPIE Event: Optical Engineering + Applications, 2007, San Diego, California, United SPIE. States 


\section{PROCEEDINGS OF SPIE}

\section{Focal Plane Arrays for Space Telescopes III}

Thomas J. Grycewicz

Cheryl J. Marshall

Penny G. Warren

Editors

27-28 August 2007

San Diego, California, USA

Sponsored and Published by

SPIE

Volume 6690 
The papers included in this volume were part of the technical conference cited on the cover and title page. Papers were selected and subject to review by the editors and conference program committee. Some conference presentations may not be available for publication. The papers published in these proceedings reflect the work and thoughts of the authors and are published herein as submitted. The publisher is not responsible for the validity of the information or for any outcomes resulting from reliance thereon.

Please use the following format to cite material from this book:

Author(s), "Title of Paper," in Focal Plane Arrays for Space Telescopes III, edited by Thomas J. Grycewicz, Cheryl J. Marshall, Penny G. Warren, Proceedings of SPIE Vol. 6690 (SPIE, Bellingham, WA, 2007) Article CID Number.

ISSN 0277-786X

ISBN 9780819468383

Published by

SPIE

P.O. Box 10, Bellingham, Washington $98227-0010$ USA

Telephone +1 3606763290 (Pacific Time) · Fax +1 3606471445

SPIE.org

Copyright (c) 2007, Society of Photo-Optical Instrumentation Engineers

Copying of material in this book for internal or personal use, or for the internal or personal use of specific clients, beyond the fair use provisions granted by the U.S. Copyright Law is authorized by SPIE subject to payment of copying fees. The Transactional Reporting Service base fee for this volume is $\$ 18.00$ per article (or portion thereof), which should be paid directly to the Copyright Clearance Center (CCC), 222 Rosewood Drive, Danvers, MA 01923. Payment may also be made electronically through CCC Online at copyright.com. Other copying for republication, resale, advertising or promotion, or any form of systematic or multiple reproduction of any material in this book is prohibited except with permission in writing from the publisher. The CCC fee code is $0277-786 \mathrm{X} / 07 / \$ 18.00$.

Printed in the United States of America.

Publication of record for individual papers is online in the SPIE Digital Library.

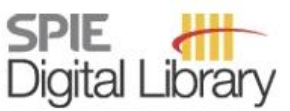

SPIEDigitalLibrary.org

Paper Numbering: Proceedings of SPIE follow an e-First publication model, with papers published first online and then in print and on CD-ROM. Papers are published as they are submitted and meet publication criteria. A unique, consistent, permanent citation identifier (CID) number is assigned to each article at the time of the first publication. Utilization of CIDs allows articles to be fully citable as soon they are published online, and connects the same identifier to all online, print, and electronic versions of the publication. SPIE uses a six-digit CID article numbering system in which:

- The first four digits correspond to the SPIE volume number.

- The last two digits indicate publication order within the volume using a Base 36 numbering system employing both numerals and letters. These two-number sets start with $00,01,02,03,04,05$, 06, 07, 08, 09, OA, OB ... 0Z, followed by 10-1Z, 20-2Z, etc.

The CID number appears on each page of the manuscript. The complete citation is used on the first page, and an abbreviated version on subsequent pages. Numbers in the index correspond to the last two digits of the six-digit CID number. 


\section{Contents}

vii Conference Committee

\section{SESSION 1 NEW DEVELOPMENTS IN SATELLITE FPAs I}

669003 Fundamental performance differences between CMOS and CCD imagers: Part II (Invited Paper) [6690-02]

J. Janesick, J. Andrews, J. Tower, M. Grygon, Sarnoff Corp. (USA); T. Elliott, Jet Propulsion Lab. (USA); J. Cheng, Chronicle Technology (USA); M. Lesser, Steward Observatory, Univ. of Arizona (USA); J. Pinter, Sarnoff Corp. (USA)

669004 Inter-pixel capacitance in fully-depleted silicon hybrid CMOS focal plane arrays [6690-03] Y. Bai, M. C. Farris, A. K. Petersen, J. W. Beletic, Teledyne Imaging Sensors (USA)

669005 Conversion gain non-linearity and its correction in hybridised near infrared detectors [6690-04]

N. Bezawada, D. Ives, D. Atkinson, UK Astronomy Technology Ctr. (United Kingdom)

669006 Comparing the low-temperature performance of megapixel NIR InGaAs and HgCdTe imager arrays [6690-05]

S. Seshadri, D. M. Cole, B. Hancock, P. Ringold, C. Peay, C. Wrigley, Jet Propulsion Lab. (USA); M. Bonati, California Institute of Technology (USA); M. G. Brown, M. Schubnell, Univ. of Michigan (USA); G. Rahmer, D. Guzman, California Institute of Technology (USA); D. Figer, Rochester Institute of Technology (USA); G. Tarle, Univ. of Michigan (USA); R. M. Smith, California Institute of Technology (USA); C. Bebek, Lawrence Berkeley National Lab. (USA)

669007 Development of a thinned back-illuminated CMOS active pixel sensor for extreme ultraviolet spectroscopy and imaging in space science (Invited Paper) [6690-06] N. Waltham, M. Prydderch, H. Mapson-Menard, M. Clapp, Rutherford Appleton Lab. (United Kingdom); P. Pool, A. Harris, e2v technologies (United Kingdom)

669008 Realization and application of a 111 million pixel backside-illuminated detector and camera [6690-08]

N. Zacharias, B. Dorland, U.S. Naval Observatory (USA); R. Bredthaver, K. Boggs,

G. Bredthaver, Semiconductor Technology Associates (USA); M. Lesser, Steward

Observatory, Univ. of Arizona (USA)

\section{SESSION 2 NEW DEVELOPMENTS IN SATELLITE FPAS II}

669009 Back-illuminated three-dimensionally integrated CMOS image sensors for scientific applications (Invited Paper) [6690-09]

V. Suntharalingam, D. D. Rathman, G. Prigozhin, S. Kissel, M. Bautz, Massachusetts Institute of Technology (USA) 
66900 A The Gaia focal plane [6690-10]

A. Laborie, R. Davancens, P. Pouny, C. Vétel, F. Chassat, P. Charvet, EADS Astrium (France);

P. Garé, G. Sarri, European Space Research and Technology Ctr. (Netherlands)

6690 OB Mission to Mars: the HiRISE camera on-board MRO (Invited Paper) [6690-11]

T. H. Ebben, J. Bergstrom, P. Spuhler, Ball Aerospace and Technologies Corp. (USA);

A. Delamere, Delamere Space Sciences (USA); D. Gallagher, CDM Optics Inc. (USA)

SESSION 3 FPA CHARACTERIZATION AND TEST

6690 OC High-performance focal plane arrays based on the HAWAll-2RG/4RG and the SIDECAR

ASIC (Invited Paper) [6690-12]

M. Loose, J. Beletic, J. Garnett, M. XU, Teledyne Imaging Sensors (USA)

6690 OD Laboratory and sky testing results for the TIS H4RG-10 4k $\times 4 \mathrm{k}$ 10-micron visible CMOShybrid detector (Invited Paper) [6690-13]

B. N. Dorland, G. S. Hennessy, N. Zacharias, D. G. Monet, H. Harris, U.S. Naval Observatory (USA); C. Rollins, Research Support Instruments, Inc. (USA); P. Shu, L. Miko, B. Mott, NASA Goddard Space Flight Ctr. (USA); A. Waczynski, E. Kan, G. Delo, Global Science and Technology, Inc. (USA)

6690 OE Radiation effects in two InGaAs focal plane arrays [6690-14]

F. Knight, M. Waldon, B. Greensmith, M. Mattei, L. Tonia, N. Gould, MIT Lincoln Lab. (USA)

6690 OF Cryogenic testing of a 1024×1024 Si:As array for WISE [6690-15]

J. L. Dotson, M. McKelvey, R. McMurray, Jr., J. Goebel, NASA Ames Research Ctr. (USA);

A. Mainzer, Jet Propulsion Lab. (USA)

$6690 \mathrm{OH} \quad$ First use of a HyViSI H4RG for astronomical observations [6690-17]

L. M. Simms, Stanford Linear Accelerator Ctr. (USA); D. F. Figer, B. J. Hanold, D. J. Kerr, Rochester Institute of Technology (USA); D. K. Gilmore, S. M. Kahn, Stanford Linear Accelerator Ctr. (USA); J. A. Tyson, Univ. of California, Davis (USA)

POSTER SESSION

6690 Ol Radiometric and noise characteristics of SI-1920HD cameras built from the AltaSens ProCamHD 3560 FPA [6690-18]

R. Kessel, W. J. Scharpf, Naval Research Lab. (USA); D. M. Huber, C. J. Rollins, Research Support Instruments, Inc. (USA); B. Dorland, G. S. Hennessy, U.S. Naval Observatory (USA); B. E. Plourde, Assurance Technology Corp. (USA)

6690 0J Astrometric sky testing results for the TIS 5-micron 3T-class CMOS detector [6690-19] B. N. Dorland, G. S. Hennessy, N. Zacharias, U.S. Naval Observatory (USA); C. Rollins,

D. Huber, Research Support Instruments (USA); R. Kessel, Naval Research Lab. (USA)

6690 OK Laboratory and radiation performance testing results for the e2v model 212 CCD [6690-20] B. N. Dorland, U.S. Naval Observatory (USA); R. Foltz, Sigma Space, Inc. (USA); A. Waczynski, Global Science and Technology, Inc. (USA) 
$6690 \mathrm{OL}$ Characterization of the detector subsystem for near-infrared spectrograph (NIRSpec) on the James Webb Space Telescope [6690-21]

D. B. Mott, NASA Goddard Space Flight Ctr. (USA); A. Waczynski, Global Science \& Technology, Inc. (USA); Y. Wen, MEl Technologies, Inc. (USA); W. Xia-Serafino, Global Science \& Technology, Inc. (USA); B. J. Rauscher, NASA Goddard Space Flight Ctr. (USA); R. J. Hill, Science Systems and Applications, Inc. (USA); G. Delo, R. Foltz, E. Kan, Global Science \& Technology, Inc. (USA); M. P. Chiao, MEl Technologies, Inc. (USA); O. Fox, Univ. of Virginia (USA); C. Cabelli, J. Garnett, M. Loose, S. Wong-Anglin, M. Zandian, Teledyne Imaging Sensors (USA); D. Alexander, Northrop Grumman Technical Services (USA); C. K. Brambora, R. Derro, NASA Goddard Space Flight Ctr. (USA); T. Ellis, ITT Space Systems LLC (USA); M. B. Garrison, NASA Goddard Space Flight Ctr. (USA); B. Howe, ITT Space Systems LLC (USA); T. E. Johnson, NASA Goddard Space Flight Ctr. (USA); M. Jurado, ITT Space Systems LLC (USA); S. S. Manthripragada, J. M. Marsh, C. Marshall, R. J. Martineau, NASA Goddard Space Flight Ctr. (USA); J. Nieznanski, ITT Space Systems LLC (USA); K. Novo-Gradac, SGT, Inc. (USA); W. D. Roher, Northrop Grumman Technical Services (USA); M. T. Smith, D. Wilson, NASA Goddard Space Flight Ctr. (USA); P. Wallis, ITT Space Systems LLC (USA)

6690 0M Detector arrays for the James Webb Space Telescope near-infrared spectrograph [6690-22]

B. J. Rauscher, D. Alexander, C. K. Brambora, R. Derro, C. Engler, NASA Goddard Space Flight Ctr. (USA); O. Fox, NASA Goddard Space Flight Ctr. (USA) and Univ. of Virginia (USA); M. B. Garrison, G. Henegar, NASA Goddard Space Flight Ctr. (USA); R. J. Hill, NASA Goddard Space Flight Ctr. (USA) and Conceptual Analytics LLC (USA); T. Johnson, NASA Goddard Space Flight Ctr. (USA); D. J. Lindler, Sigma Space Corp. (USA);

S. S. Manthripragada, C. Marshall, B. Mott, T. M. Parr, NASA Goddard Space Flight Ctr. (USA); W. D. Roher, NASA Goddard Space Flight Ctr. (USA) and Northrop Grumman Technical Services (USA); K. B. Shakoorzadeh, NASA Goddard Space Flight Ctr. (USA) and AK Aerospace Technology Corp. (USA); M. Smith, NASA Goddard Space Flight Ctr. (USA); A. Waczynski, NASA Goddard Space Flight Ctr. (USA) and Global Science and Technologies, Inc. (USA); Y. Wen, NASA Goddard Space Flight Ctr. (USA) and Muniz Engineering Inc. (USA); D. Wilson, NASA Goddard Space Flight Ctr. (USA); W. Xia-Serafino, NASA Goddard Space Flight Ctr. (USA) and Global Science and Technologies, Inc. (USA); C. Cabelli, Teledyne Imaging Sensors (USA); E. Cheng, Teledyne Imaging Sensors (USA) and Conceptual Analytics LLC (USA); J. Garnett, M. Loose, M. Zandian, J. Zino, Teledyne Imaging Sensors (USA); T. Ellis, B. Howe, M. Jurado, G. Lee, J. Nieznanski, P. Wallis, J. York, ITT Space Systems Division (USA); M. W. Regan, Space Telescope Science Institute (USA); G. Bagnasco, T. Böker, G. De Marchi, ESTEC (Netherlands); P. Ferruit, Univ. de Lyon (France), Univ. Lyon 1 (France), and CNRS, Ecole Normale Supérieure de Lyon (France); P. Jakobsen, P. Strada, ESTEC (Netherlands)

Author Index 
Downloaded From: https://www.spiedigitallibrary.org/conference-proceedings-of-spie on 26 Apr 2023

Terms of Use: https://www.spiedigitallibrary.org/terms-of-use 


\title{
Conference Committee
}

\author{
Conference Chairs \\ Thomas J. Grycewicz, The Aerospace Corporation (USA) \\ Cheryl J. Marshall, NASA Goddard Space Flight Center (USA) \\ Penny G. Warren, Ball Aerospace \& Technologies Corporation (USA) \\ Program Committee \\ Sachi Babu, NASA Goddard Space Flight Center (USA) \\ James W. Beletic, Teledyne Imaging Sensors (USA) \\ Richard A. Bredthauer, Semiconductor Technology Associates Inc. \\ (USA) \\ Mark C. Clampin, NASA Goddard Space Flight Center (USA) \\ Michael P. Lesser, Steward Observatory, The University of Arizona (USA) \\ Terrence S. Lomheim, The Aerospace Corporation (USA) \\ Kyle B. Miller, Ball Aerospace \& Technologies Corporation (USA) \\ Peter J. Pool, e2v technologies Ltd. (United Kingdom) \\ John Traylor, Naval Research Laboratory (USA)
}

Session Chairs

1 New Developments in Satellite FPAs I

Bernard J. Rauscher, NASA Goddard Space Flight Center (USA)

2 New Developments in Satellite FPAs II

Bernard J. Rauscher, NASA Goddard Space Flight Center (USA)

3 FPA Characterization and Test

Mark E. McKelvey, NASA Ames Research Center (USA) 
Downloaded From: https://www.spiedigitallibrary.org/conference-proceedings-of-spie on 26 Apr 2023

Terms of Use: https://www.spiedigitallibrary.org/terms-of-use 